\title{
Circulatory pattern of cytokines, adipokines and bone markers in postmenopausal women with low BMD
}

This article was published in the following Dove Press journal:

Journal of Inflammation Research

\author{
Fawaz Y Azizieh' \\ Diaa Shehab ${ }^{2}$ \\ Khaled Al Jarallah ${ }^{2}$ \\ Olusegun Mojiminiyi ${ }^{3}$ \\ Renu Gupta ${ }^{4}$ \\ Raj Raghupathy ${ }^{5}$ \\ 'Department of Mathematics and Natural \\ Sciences, International Centre for \\ Applied Mathematics and Computational \\ Bioengineering, Gulf University for \\ Science and Technology, Kuwait City, \\ Kuwait; ${ }^{2}$ Department of Medicine, \\ Faculty of Medicine, Kuwait University, \\ Kuwait City, Kuwait; ${ }^{3}$ Department of \\ Pathology, Faculty of Medicine, Kuwait \\ University, Kuwait City, Kuwait; \\ ${ }^{4}$ Department of Radiology, Faculty of \\ Medicine, Kuwait University, Kuwait City, \\ Kuwait; ${ }^{5}$ Department of Microbiology, \\ Faculty of Medicine, Kuwait University, \\ Kuwait City, Kuwait
}

Correspondence: Fawaz Y Azizieh

Department of Mathematics and Natural Sciences, Gulf University for Science and Technology, Block 5, Building I Mubarak Al-Abdullah Area/West Mishref, Kuwait City, Kuwait

Tel +96525307499

Email Azizieh.F@gust.edu.kw
Objective: In addition to some well-characterized bone turnover markers (BTMs), cytokines and adipokines have also been suggested to be linked to osteoporosis seen in menopause. However, there is much controversy on the possible association between these markers and bone mineral density (BMD). This study was aimed at measuring circulatory levels of selected cytokines, adipokines and BTMs in postmenopausal women with normal and low BMD.

Methods: The study population included 71 post-menopausal women, of whom 25 had normal BMD, 31 had osteopenia and 13 had osteoporosis. Circulatory levels of selected proresorptive (TNF- $\alpha$, IL-1 $\beta$, IL-6, IL-8, IL-12, IL-17), anti-resorptive (IFN- $\gamma$, IL-4, IL-10, IL13 , TGF- $\beta$ ) and five adipokine markers (adiponectin, adipsin, lipocalin-2/NGAL, PAI-1 and resistin) were measured using the Multiplex system and read on the Magpix ELISA platform. Further, two bone turnover markers (PINP, CTX) as well as estradiol levels were assayed from the same samples.

Results: While circulatory levels of cytokines were comparable between groups, women with low BMD had statistically significantly higher median circulatory levels of adipokines as compared to those with normal BMD. Further, while levels of CTX were not different between the two groups; PINP, PINP/CTX ratio and estradiol levels were significantly lower in women with low BMD. Levels of adiponectin, PINP, PINP/CTX ratio and estradiol correlated significantly with BMD of the hip and spine.

Conclusion: The associations between various markers and BMD are complex and multivariate. Our data provide insights into the possible use of circulatory levels of cytokines, adipokines and bone turnover markers on the pathogenesis of postmenopausal osteoporosis because of the well-documented effects of these molecules on bone tissue and their relevance to osteoporosis.

Keywords: cytokines, adipokines, BTM, osteoporosis, menopause

\section{Introduction}

Osteoporosis is among the most important conditions associated with aging. ${ }^{1}$ With the increase in life expectancy, osteoporosis is becoming a major worldwide health problem. Clinically, osteoporosis is a skeletal disorder characterized by compromised bone strength predisposing a person to an increased risk of fracture. On the cellular level, research is focusing on factors that may reduce the activity of osteoclasts and/or increase the activity of osteoblasts thus reducing bone turnover. This continues to encourage research activities on elucidating the pathophysiology 
of the osteoporotic processes, with the objective of modulating or reversing these processes.

While both men and women are affected by aging osteoporosis, the incidence in women is higher. According to recent statistics from the International Osteoporosis Foundation, worldwide, 1 in 3 women over the age of 50 years and 1 in 5 men will experience osteoporotic fractures in their lifetime. Therefore, postmenopausal osteoporosis continuous to pose a significant challenge with a great deal of controversy related to contributing factors and markers.

The immune system is now understood to contribute to postmenopausal osteoporosis possibly through the involvement of cytokines that modulate osteoblast and osteoclast activity. ${ }^{2-4}$ Several pro-inflammatory cytokines have been implicated in the regulation of osteoblasts and osteoclasts, and a shift towards an activated immune profile has been hypothesized to be an important risk factor. ${ }^{5,6}$ Studies related to cytokine balance in human osteoporosis are still few in number, and the majority of data have been derived from animal models and cellular studies. There is a need to study circulatory plasma levels of cytokines in postmenopausal women with normal or low BMD and study if these can be used as potential markers of the disease.

In addition to the immune system, it has further been revealed that the adipose tissue has independent endocrine and paracrine activity associated with the production of many bioactive molecules, known as adipokines, that influence metabolic processes, regulate bone metabolism and may be involved in the pathophysiology of osteoporosis. ${ }^{7,8}$ Adiponectin is one of the adipokines that is highly expressed in visceral fat, abundantly present in the circulation and believed to influence bone metabolism. ${ }^{8,9}$ Leptin is released by adipose tissue and reported to enhance osteoblast differentiation and to inhibit osteoclast generation; however, its effects on bone metabolism are controversial. ${ }^{10}$ The effects of resistin and other adipokines are still under study. While only a few cross-sectional studies have focused on the association between serum adipokines and bone turnover/remodeling in humans, many clinical studies have questioned the association between serum adiponkines levels and bone mineral density (BMD). ${ }^{11-13}$

While cytokines and adipokines are relatively recent, many other chemical bone turnover markers (BTMs) have been studied extensively in metabolic bone diseases, including osteoporosis. This explains the wide range of biochemical markers that have been developed. ${ }^{14}$ However, despite extensive research, uncertainties remain over their use in routine clinical practice to assess risk of fracture and/or monitor treatment. ${ }^{15-17}$ Limitations include their biological variability and the multiple methodologies used for the quantification of the same analytes.

A working group of the International Osteoporosis Foundation (IOF) and the International Federation of Clinical Chemistry and Laboratory Medicine (IFCC) has recently recommended the use of one bone formation marker (procollagen type I N propeptide [PINP]) and one bone resorption marker (C-terminal cross-linking telopeptide of type I collagen [CTX]) as reference markers in future studies on fracture risk assessment so that sufficient data are accumulated for the assessment of their potential for inclusion in fracture risk-assessment tools. ${ }^{17}$ In order to use these bone turnover markers for harmonized clinical purposes, it was further considered necessary to establish reference intervals for different geographic areas and ethnicities. ${ }^{17}$ Furthermore, due to differences that currently exist between results from different commercial clinical assays, current reference intervals need to be methodspecific, as reference intervals from different methods cannot be used interchangeably. Currently, there are three clinical assays available for PINP and for CTX in blood. For PINP, these are the automated Roche Elecsys 2010 assay which measures total PINP, the radioimmunoassay for intact PINP (Orion Diagnostica UniQ PINP) and the IDS iSYS Intact PINP assay. The three commercial assays to measure plasma CTX are the IDS iSYS assay, the IDS ELISA and Roche methods. Several metanalysis studies are looking into the compatibility of PINP and CTX values generated by these different clinical assays. ${ }^{18}$ In terms of sample type, EDTA plasma has been reported to be the preferred specimen type for the assay of CTX and is identified as such when specific reference is made. PINP was found to be less affected by specimen type. ${ }^{17}$

In summary, there is continued support for the potential for BTMs to provide clinically useful information in spite of the many limitations identified. The IOF-IFCC Bone Marker Standards Working Group ${ }^{17}$ recommended that future clinical studies should focus on using standardized analytical methods of reference analytes. Data from the Middle East Gulf region are of substantial value to the international efforts.

On the other hand, no one can overlook the tight concomitant decline of ovarian function and bone density. As a result, the estrogen-centric paradigm of postmenopausal osteoporosis has dominated for years. However, with the serious shortcomings of estrogen therapies, current research on the pathogenesis of osteoporosis has moved from the 
traditional context of a single disease entity, to comorbidities that accompany old age. ${ }^{19-21}$ As estrogen was the most intensively investigated, an impressive number of studies revealed its fundamental role in maintaining bone density. $^{22-26}$ Estrogen suppresses the differentiation and enhances apoptosis of osteoclasts, enhances osteoblast differentiation and increases their life span by decreasing apoptosis, therefore reducing bone turnover. ${ }^{27}$ While these effects are at least in part mediated directly through highaffinity estrogen receptors present on bone cells, it is now becoming clear that the effect of estrogen is through other pathways. Estrogen suppresses the production of proresorptive cytokines such as tumour necrosis factor-alpha (TNF- $\alpha$ ), interleukin (IL)-1, IL-8, IL-6, IL-17 as well as the receptor activator of nuclear factor $\kappa \mathrm{B}$ ligand (RANKL), while on the contrary, it has been shown to increase the production of anti-resorptive ones such as tumor growth factor-beta (TGF- $\beta$ ), interferon-gamma (IFN- $\gamma$ ) as well as the production of osteoprotegrin $(\mathrm{OPG})$. $^{3,28}$

Nevertheless, it remains unknown whether, and to what extent, small differences in levels of estrogen in the circulation contribute to changes of cytokines, adipokines, bone turnover markers or BMD in postmenopausal women. This emphasizes the need for further studies on the levels of estrogen in postmenopausal women. Thus, this study is aimed at exploring this further.

This research work is an additional effort along these lines looking to further our understanding of the association between selected circulatory markers and postmenopausal osteoporosis.

\section{Methodology Patient selection}

We studied 71 women who were postmenopausal (ie, absence of menstrual periods for at least 12 months prior to the study). All participants were recruited consecutively from the Physical Medicine Unit at Mubarak Al Kabeer Hospital, Kuwait, and underwent clinical assessment by a single recruiter. These were grouped as women with normal BMD $(\mathrm{N}, \mathrm{n}=25)$ or with low BMD (L, $\mathrm{n}=46)$. Participants were further grouped by the T-scores of BMD into three groups: the normal group $(\mathrm{N}, \mathrm{T}$-scores $\geq-1, \mathrm{n}=25$ ), the osteopenia group (OSN, $-2.5<$ T-scores $<$ $-1, n=31)$ and the osteoporosis group (OSR, T-scores $\leq-$ $2.5, \mathrm{n}=15)$. This was based on the guidelines of the WHO and Adult Official Positions of the International Society for Clinical Densitometry (ISCD) (http://www.iscd.org/offi cial-positions/2015-iscd-official-positions-adult/) as updated in 2015. All participants were interviewed, evaluated clinically and demographic data such as age, weight, height, body mass index (BMI) and period since menopause recorded on the day of examination. Women who were on systemic corticosteroids, or had malignancy, hyperparathyroidism, severe renal impairment, liver disease or infectious disease were excluded due to possible effects on immune function and cytokine production. Women who needed calcium and vitamin D supplementation were receiving $600 \mathrm{mg}$ of calcium and 200 IU of vitamin D twice daily. This study was granted ethical approval from the Health Sciences Center of Kuwait University, and all participants gave written informed consent prior to participating in the study.

\section{BMD measurement}

Total body measurements of BMD and bone mineral content (BMC) were measured using dual-energy X-ray absorptiometry (GE Medical System Lunar; Madison, WI). Total BMD and BMC were measured with a precision (coefficient of variation) of $0.7 \%$. BMD was measured at total lumbar spine (L1-L4) and left hip. The diagnosis was done by a single consultant interpreter using T-score values according to the criteria set by WHO as mentioned above.

\section{Blood sampling and sample storage}

The procedures for blood sampling and transport were similar for all subjects. Venous blood samples taken in the early morning were collected into two types of vacutainer tubes: serum separation tubes (SST) - allowed to clot at room temperature for $30 \mathrm{mins}$, and EDTA vacutainer tubes. Both tubes were then centrifuged for $10 \mathrm{mins}$ at $3,000 \times \mathrm{g}$ and sera/plasma samples were stored at $-20^{\circ} \mathrm{C}$ till analysis.

\section{Measurement of circulatory cytokines and adipokines levels}

Plasma levels of pro-resorptive stimulator cytokines TNF$\alpha$, IL-1 $\beta$, IL-6, IL-8, IL-12, IL-17 and anti-resorptive inhibitor cytokines IFN- $\gamma$, IL-4, IL-10, IL-13, GMCSF, TGF- $\beta$ were measured from all groups using the MILLIPLEX ${ }^{\circledR}$ MAP HCYP2MAG-62K Human Cytokine Magnetic Bead Panel. The sensitivity (minimum detectable concentrations, $\mathrm{pg} / \mathrm{mL}$ ) of the assays were as follows: TNF- $\alpha$ : 0.7 , IL-1 $\beta$ : 0.8, IL-6: 0.9, IL-8: 0.4, IL-12: 0.6; IL-17: 0.6, 
IFN- $\gamma: 0.7$, IL-4: 4.5, IL-10: 1.1; IL-13: 1.3; GMCSF: 7.5; TGF- $\beta$ : 1.5 .

Serum levels of adipokines were measured using the MILLIPLEX® MAP HADK1MAG-61K adipokine magnetic bead panel that includes: adiponectin, adipsin, lipocalin-2/NGAL (neutrophil gelatinase-associated lipocalin), PAI-1 (plasminogen activator inhibitor-1) and resistin. The sensitivity of each of the assays (minimum detectable concentrations, $\mathrm{pg} / \mathrm{mL}$ ) was as follows: adiponectin: 1.1 , adipsin: 4.8, lipocalin-2/NGAL: 1.7, PAI-1:4.1, resistin: 2.2 .

Concentrations of all analytes were determined using a Magpix Array Reader (Luminex, Austin, TX) that quantitates multiplex immunoassays in a 96-well format with very small sample volumes. Analyte concentrations were calculated using a standard curve, with software provided by the manufacturer (Luminex Manager Software). Quality control measures were followed as per the recommendations of the manufacturer. The intra- and inter-assay CVs for all parameters tested were $<10 \%$ and $<15 \%$, respectively.

\section{Circulatory levels of PINP, $\beta$-ctx and estradiol}

As per the recommendation of the IOF and IFCC, ${ }^{17}$ Roche assays were used to quantitate total procollagen type I amino-terminal propeptide (PINP) in serum samples (Cat. \# 03141071 190) and $\beta$-CrossLaps/serum ( $\beta$-CTX) levels in EDTA plasma samples (Cat. \# 11,972,308 122). Levels of $17 \beta$ estradiol, which represent $90 \%$ of all estrogens, were as well measured from serum samples using Roche assays (Cat \# 06656021 190). All assays were run on Elecsys 2010 (Roche Diagnostics). Manufacturer's instructions were followed for all parameters. The sensitivity (minimum detectable concentrations) of the assays were as follows: PINP: $5 \mathrm{ng} / \mathrm{mL}$, CTX: $0.01 \mathrm{ng} / \mathrm{mL}(10$ $\mathrm{pg} / \mathrm{mL}$ ), estradiol: $5 \mathrm{pg} / \mathrm{mL}$.

\section{Statistical analysis}

Statistical analysis was done using the SPSS version 23 software (SPSS Inc., Chicago, IL, USA). Normality distribution of data was first determined by the KolmogorovSmirnov test and accordingly, groups were compared using one-way ANOVA, Student's T-test or MannWhitney U test. Categorical variables were compared using Pearson's Chi-square test. Spearman's rank correlation coefficient was calculated to determine correlations between different measures and cytokine levels. A $P$-value of less than or equal to 0.05 was considered statistically significant for all tests.

We further used regression models to check if the subjects' age or years since menopause were potential confounders in the search for possible associations between BMD groups and different markers, particularly as these variables were different between the two groups. Given the binary outcome variable (two groups; normal vs low BMD) we used logistic regression models to ascertain whether these variables could be potential confounders. We first fitted an unadjusted crude model with each of the markers followed by age or years since menopauseadjusted models. We did not have information on other potential confounders to consider.

\section{Results}

Among the 71 postmenopausal women, $64.7 \%$ had low BMD ( $L, n=46)$; although these women were older in age as compared to women with normal BMD ( $N, n=25)$ $(p=0.03)$, there was no significant difference in terms of the number of years since menopause. Moreover, weight, height and body mass index (BMI) were comparable between the two groups (Table 1).

While women with normal BMD (N, n=25) and osteopenia (OSN, $\mathrm{n}=31$ ) had comparable age and years since menopause, women with osteoporosis (OSR, $n=15)$ were significantly older than the normal BMD group $(p=0.001)$ and had a longer duration since menopause as compared to women with normal and osteopenic BMD (0.06 and 0.019 , respectively). Weight, height, BMI, smoking and previous fracture history were also comparable between the groups (Table 1).

\section{Circulatory levels of cytokines in postmenopausal women with normal or low BMD}

Levels of IL-1 $\beta$ and GMCSF were not detected in any of the sera samples. Circulatory cytokine patterns of both pro-resorptive (TNF- $\alpha$, IL-6, IL-8, IL-12, IL-17) and antiresorptive (IFN- $\gamma$, IL-4, IL-10, IL-13, TGF- $\beta$ ) were similar in all groups. The only exception was for TGF- $\beta$ where its circulatory levels were significantly higher in osteopenic as compared to osteoporotic women (Table 2).

Further, there were no significant correlations between the circulatory levels of any of the cytokines tested and BMD of the hip or spine. 
Table I Demographic data and baseline clinical characteristics of postmenopausal women enrolled in the study (mean \pm SD)

\begin{tabular}{|c|c|c|c|c|c|c|c|c|}
\hline & $\begin{array}{l}\text { Normal } \\
\text { BMD }\end{array}$ & $\begin{array}{l}\text { Low } \\
\text { BMD }\end{array}$ & $p$ & Osteopenia & $p$ & Osteoporosis & $\mathbf{p}$ & $p$ \\
\hline & $\begin{array}{l}(N) \\
(n=25)\end{array}$ & $\begin{array}{l}(L) \\
(n=46)\end{array}$ & (N vs $L)$ & $\begin{array}{l}(\text { OSN }) \\
(n=3 I)\end{array}$ & $\begin{array}{l}\text { (N vs } \\
\text { OSN) }\end{array}$ & $\begin{array}{l}\text { (OSR) } \\
(n=\mid 5)\end{array}$ & $\begin{array}{l}\text { (N vs } \\
\text { OSR) }\end{array}$ & $\begin{array}{l}\text { (OSN vs } \\
\text { OSR) }\end{array}$ \\
\hline Age (years) & $56.1 \pm 5.6$ & $59.6 \pm 7.8$ & 0.03 & $58.7 \pm 7.9$ & 0.16 & $61.3 \pm 7.3$ & 0.001 & 0.14 \\
\hline Weight (Kg) & $80.5 \pm 12.2$ & $75.5 \pm 12.7$ & 0.13 & $75.7 \pm 12.1$ & 0.55 & $75.2 \pm 12.3$ & 0.18 & 0.96 \\
\hline Height (m) & $158.7 \pm 5.2$ & $156.1 \pm 6.1$ & 0.15 & $157.3 \pm 5.3$ & 0.18 & $153.5 \pm 6.9$ & 0.02 & 0.58 \\
\hline BMI $\left(\mathrm{kg} / \mathrm{m}^{2}\right)$ & $32.0 \pm 5.2$ & $31.0 \pm 5.0$ & 0.47 & $30.6 \pm 5.0$ & 0.28 & $31.8 \pm 5.1$ & 0.90 & 0.40 \\
\hline Smoking (\%) & $2(8)$ & $2(4)$ & 0.62 & $2(6.5)$ & 1.00 & $0(0)$ & 0.52 & 0.55 \\
\hline Previous fracture (\%) & $4(16)$ & $10(21.7)$ & 0.55 & $7(22.6)$ & 0.62 & $3(20)$ & 0.69 & 1.00 \\
\hline $\begin{array}{l}\text { Years since meno- } \\
\text { pause }(y r)\end{array}$ & $7.6 \pm 5.5$ & $9.0 \pm 7.2$ & 0.53 & $7.4 \pm 6.4$ & 0.75 & $12.1 \pm 7.7$ & 0.06 & 0.019 \\
\hline T-score hip & $0.2 \pm 0.8$ & $-1.4 \pm 0.87$ & 0.0001 & $-1.1 \pm 0.8$ & $0.000 \mathrm{I}$ & $-2.0 \pm 0.7$ & 0.0001 & 0.001 \\
\hline Hip BMC $\left(\mathrm{g} / \mathrm{cm}^{2}\right)$ & $1.02 \pm 0.12$ & $0.78 \pm 0.12$ & 0.0001 & $0.8 I \pm 0.13$ & $0.000 \mathrm{I}$ & $0.73 \pm 0.1$ & 0.0001 & 0.074 \\
\hline T-score LI-L4 & $-0.11 \pm 0.65$ & $-2 \pm 0.65$ & 0.0001 & $-1.7 \pm 0.4$ & 0.0001 & $-2.7 \pm 0.5$ & 0.0001 & 0.0001 \\
\hline LI-L4 BMC $\left(\mathrm{g} / \mathrm{cm}^{2}\right)$ & $1.17 \pm 0.1$ & $0.90 \pm 0.1$ & 0.0001 & $0.93 \pm 0.09$ & 0.0001 & $0.83 \pm 0.9$ & 0.0001 & 0.003 \\
\hline
\end{tabular}

Note: Significant $p$-values are depicted in bold.

Abbreviations: N, Normal BMD, L, Low BMD; OSN, Osteopenia; OSR, Osteoporosis; BMD, Bone mineral density.

Table 2 Median circulatory levels of cytokines in all groups

\begin{tabular}{|c|c|c|c|c|c|c|c|c|c|c|c|c|}
\hline \multirow{3}{*}{$\begin{array}{l}\text { Cytokine } \\
\mathrm{pg} / \mathrm{ml}\end{array}$} & \multirow{2}{*}{\multicolumn{2}{|c|}{$\begin{array}{l}\text { Normal BMD } \\
(N)(n=25)\end{array}$}} & \multirow{2}{*}{\multicolumn{2}{|c|}{$\frac{\text { Low BMD }}{(L)(n=46)}$}} & \multirow{3}{*}{$\begin{array}{l}p \\
(N \text { vs } L)\end{array}$} & \multirow{2}{*}{\multicolumn{2}{|c|}{$\begin{array}{l}\text { Osteopenia } \\
(\text { OSN) (n=3I) }\end{array}$}} & \multirow{3}{*}{$\begin{array}{l}\text { P } \\
\text { (N vs } \\
\text { OSR) }\end{array}$} & \multirow{2}{*}{\multicolumn{2}{|c|}{$\begin{array}{l}\text { Osteoporosis } \\
\text { (OSR) (n=15) }\end{array}$}} & \multirow{3}{*}{$\begin{array}{l}\text { P } \\
\text { (N vs } \\
\text { OSR) }\end{array}$} & \multirow{3}{*}{$\begin{array}{l}P \\
\text { OSN vs } \\
\text { OSR }\end{array}$} \\
\hline & & & & & & & & & & & & \\
\hline & Median & IQR & Median & IQR & & Median & IQR & & Median & IQR & & \\
\hline \multicolumn{13}{|c|}{ Pro-resorptive } \\
\hline TNF- $\alpha$ & $14 \mid 2.7$ & 2627.8 & 1711.3 & 2070.1 & 0.60 & 1601.5 & 1627.7 & 0.73 & 1963.9 & 2440.7 & 0.54 & 0.59 \\
\hline IL-6 & 4536.2 & 2710.1 & 5111.8 & 4688.8 & 0.66 & 5082.2 & 5089.9 & 0.80 & 5601.2 & 4848.6 & 0.57 & 0.72 \\
\hline IL-8 & 4.7 & 3.2 & 4.9 & 12.8 & 0.65 & 4.6 & 3.8 & 0.57 & 5.8 & 4.3 & 0.95 & 0.74 \\
\hline IL-12 & 10.2 & 11.6 & 9.9 & 12.5 & 0.82 & 10.3 & 15.2 & 0.61 & 9.8 & 12.0 & 0.73 & 0.37 \\
\hline IL-17 & 171.2 & 116.5 & 129.4 & 135.9 & 0.44 & 136.6 & 229.5 & 0.80 & 118.9 & 117.5 & 0.18 & 0.29 \\
\hline \multicolumn{13}{|c|}{ Anti-resorptive } \\
\hline $\mathrm{IFN}-\gamma$ & 420.4 & 708.9 & 469.3 & 1693.9 & 0.42 & 492.3 & 2054.2 & 0.33 & 364.9 & 1573.9 & 0.84 & 0.58 \\
\hline IL-4 & 205.6 & 497.6 & 150.9 & 243.6 & 0.61 & 188.6 & 266.7 & 0.99 & 130.4 & 202.7 & 0.25 & 0.13 \\
\hline IL-IO & 1640.9 & 3103.7 & 1826.6 & 1258.0 & 0.86 & 1954.7 & 1238.1 & 0.89 & 1644.4 & 1182.8 & 0.87 & 0.82 \\
\hline IL-I3 & $|88|$. & 755.9 & 231.8 & 397.6 & 0.93 & 298.4 & 434.0 & 0.79 & 182.9 & 350.6 & 0.52 & 0.27 \\
\hline TGF- $\beta$ & 119.4 & $|4| .8$ & 157.6 & 187.9 & 0.68 & 200.3 & 208.2 & 0.30 & 76.1 & 139.1 & 0.41 & $0.04 I$ \\
\hline
\end{tabular}

Note: Significant $p$-values are depicted in bold.

Abbreviations: N, Normal BMD, L, Low BMD; OSN, Osteopenia; OSR, Osteoporosis; IQR, Intra quartile range; BMD, Bone mineral density

\section{Circulatory levels of adipokines} (adiponectin, adipsin, lipocalin-2/NGAL, PAI-I and resistin) in sera of postmenopausal women with normal or low BMD

Serum levels of adiponectin, adipsin, PAI-1 and resistin were found significantly higher in postmenopausal women with low BMD group as compared to women with normal $\operatorname{BMD}(p=0.0001,0.034,0.032$ and 0.041 , respectively). However, levels of lipocalin-2/NGAL were not significantly different between the two groups (Figure 1).

Similarly, as compared to women with normal BMD, osteopenic women had higher levels of adiponectin, adipsin, PAI- 1 and resistin $(p=0.001,0.015,0.025$ and 0.011 , respectively). Furthermore, as compared to women with 


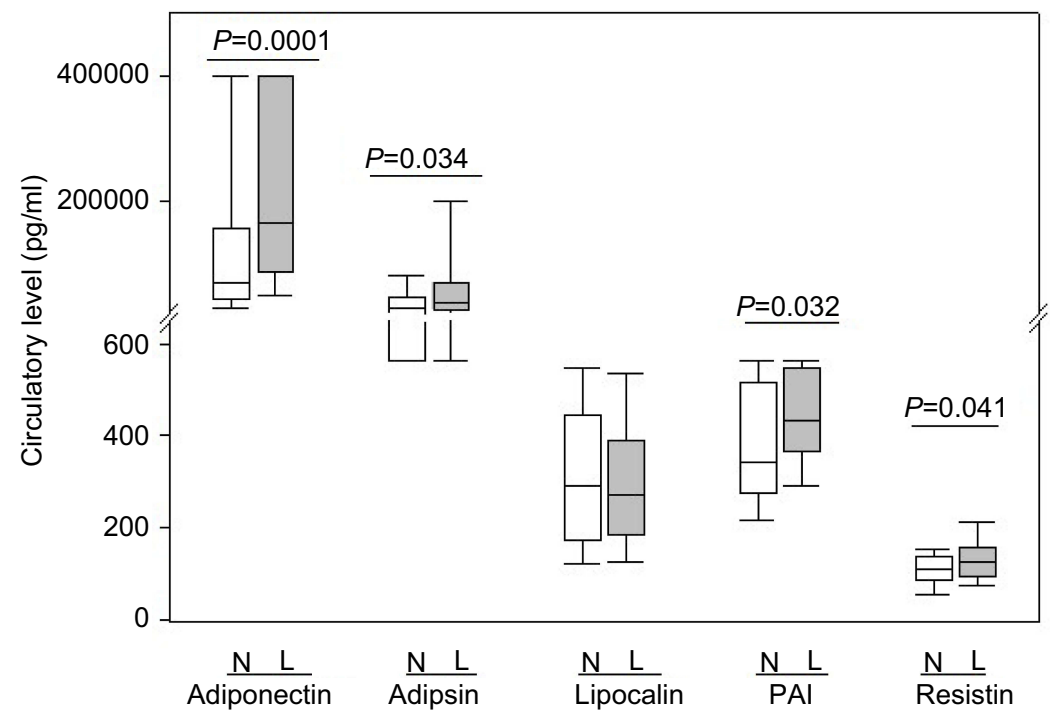

Figure I Median serum levels of adipokines in postmenopausal women with normal (N) and low BMD (L). Abbreviation: PAI, plasminogen activator inhibitor-I.

normal BMD, osteoporotic women serum levels of adiponectin were significantly higher $(p=0.015)$, while other markers were not significantly different (data not shown).

Among all the five adipokine markers measured, only adiponectin levels showed significant correlation with BMD of the hip and spine $(\mathrm{r}=-0.28,-0.27 ; p=0.038$, 0.05 , respectively).

\section{Circulatory levels of PINP, CTX and estradiol in postmenopausal women with normal or low BMD}

Median level of circulatory PINP was found significantly higher in postmenopausal women with normal BMD group as compared to women with low $\mathrm{BMD}(p=0.005)$. While median levels of CTX were comparable, levels of estradiol as well as the ratio of PINP/CTX were significantly higher in postmenopausal women with normal BMD group as compared to women with low BMD ( $p=0.0001$ and 0.026 , respectively) (Figure 2).

As compared to women with normal BMD, osteopenic women had lower levels of PINP, PINP/CTX ratio and estradiol ( $p=0.034,0.036$ and 0.003 , respectively). Furthermore, as compared to women with normal BMD, osteoporotic women had lower levels of PINP and estradiol ( $p=0.007$ and 0.0001 , respectively). However, osteopenic and osteoporotic women had comparable circulatory levels of PINP, CTX but the latter had significantly lower levels of estradiol $(p=0.008)$ (data not shown).
In terms of correlations, circulatory levels of PINP correlated significantly with CTX and estradiol levels $(\mathrm{r}=0.72,0.45 ; p=0.0001,0.0001$, respectively), as well as with BMD of the hip and spine $(\mathrm{r}=0.35,0.35$; $p=0.009,0.010$, respectively). While CTX levels did not show any correlation, the PINP/CTX ratio correlated with BMD of the hip and spine $(\mathrm{r}=0.44,0.36 ; p=0.002$, 0.013 , respectively). Similarly, estradiol levels significantly correlated with BMD of the hip and spine $(\mathrm{r}=0.42,0.32 ; p=0.001,0.017$, respectively). Other correlations were not significant.

\section{Regression analysis of bone and adipokines markers and BMD}

Table 3 presents the regression analysis of bone markers (PINP, PINP/CTX ratio), adipokines markers (adiponectin, adipsin, PA-1 and resistin) and BMD groups in unadjusted, age and years since menopause-adjusted models. The data presented are limited to markers that were significantly different between the two groups (normal vs low BMD). Factors were considered confounders if the change in the odds ratio (OR), as compared to the crude model, is $>20 \%$.

It is noteworthy that the OR values of circulatory levels of bone markers (PINP, PINP/CTX ratio) as well as adipokines markers (adiponectin, adipsin, PA-1 and resistin) were not affected by patients' age. However, all markers, except adiponectin, were affected by years since menopause indicating that it is a confounding factor (Table 3). 


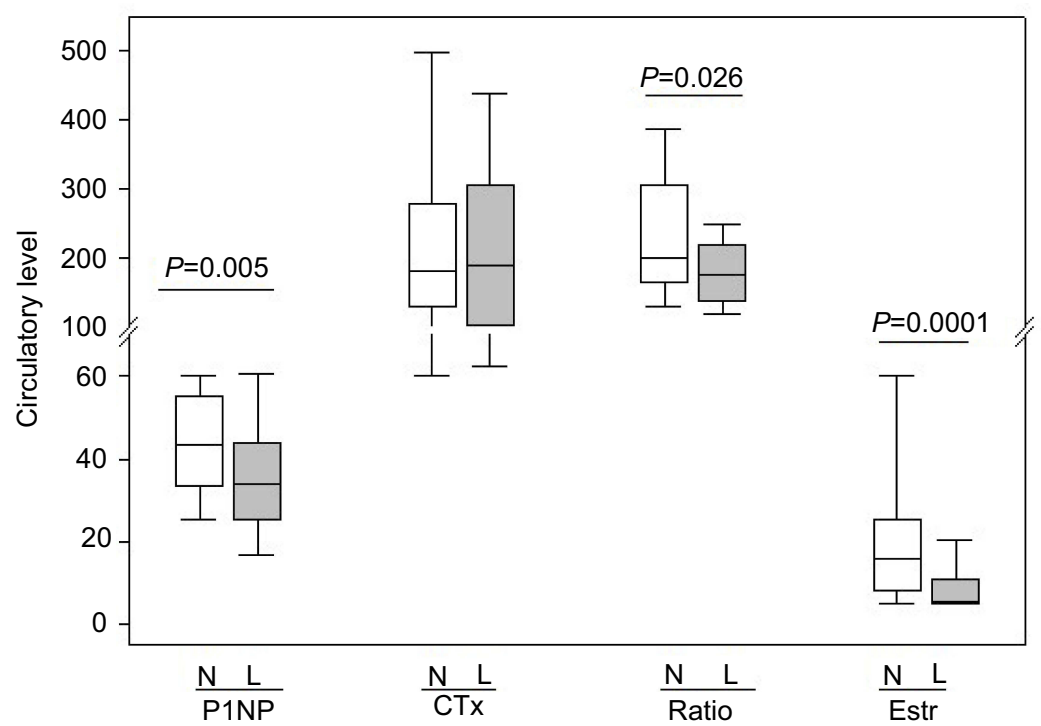

Figure 2 Median circulatory levels of PINP, CTX and estradiol in postmenopausal women with normal (N) and low BMD (L).

Abbreviations: PINP, Total procollagen type I amino-terminal propeptide (ng/ml); CTX, $\boldsymbol{\beta}$-CrossLaps (pg/ml); Ratio, PINP (ng/ml)/CTX (ng/ml); Estr, I7及 estradiol (pg/ml).

Table 3 Regression analysis of circulatory levels of bone and adipokines markers and BMD grouping in unadjusted, age-adjusted and years since menopause-adjusted models

\begin{tabular}{|c|c|c|c|c|c|c|c|}
\hline \multicolumn{2}{|c|}{ Variable (In) } & \multicolumn{2}{|c|}{ Bone Markers } & \multicolumn{4}{|c|}{ Adipokines markers } \\
\hline & & PINP & P/C ratio & Adiponectin & Adipsin & PAI-I & Resistin \\
\hline \multicolumn{8}{|l|}{ Crude } \\
\hline OR & & 0.182 & 0.146 & 2.45 & 2.03 & 3.76 & 3.87 \\
\hline \multirow{3}{*}{$\begin{array}{l}95 \% \mathrm{Cl} \\
p \text {-value }\end{array}$} & $\mathrm{LCL}$ & 0.055 & 0.033 & 1.46 & 1.11 & 1.14 & 1.16 \\
\hline & UCL & 0.606 & 0.646 & 4.09 & 3.72 & 12.37 & 12.93 \\
\hline & & 0.005 & 0.011 & 0.001 & 0.022 & 0.029 & 0.028 \\
\hline \multicolumn{8}{|c|}{ Age-adjusted model } \\
\hline OR & & 0.208 & 0.171 & 2.45 & 1.98 & 4.3 & 3.57 \\
\hline \multirow[t]{2}{*}{$95 \% \mathrm{Cl}$} & $\mathrm{LCL}$ & 0.059 & 0.037 & 1.45 & 1.05 & 1.24 & 1.06 \\
\hline & UCL & 0.732 & 0.779 & 4.15 & 3.75 & 14.88 & 12.07 \\
\hline$p$-value & & 0.014 & 0.022 & 0.001 & 0.035 & 0.021 & 0.04 \\
\hline \multicolumn{8}{|c|}{ Years since menopause-adjusted model } \\
\hline OR & & 0.3 & 0.164 & 2.71 & 1.62 & 2.37 & 3.07 \\
\hline \multirow[t]{2}{*}{$95 \% \mathrm{Cl}$} & $\mathrm{LCL}$ & 0.082 & 0.033 & 1.44 & 0.82 & 0.564 & 0.71 \\
\hline & UCL & 1.09 & 0.826 & 5.11 & 3.21 & 9.93 & 13.29 \\
\hline$p$-value & & 0.069 & 0.028 & 0.002 & 0.168 & 0.240 & 0.134 \\
\hline
\end{tabular}

Abbreviations: BMD, bone mineral density; PINP, Total procollagen type I amino-terminal propeptide (ng/ml); CTX, $\beta$-CrossLaps (pg/ml); P/C Ratio, PINP (ng/ml)/CTX (ng/ml); OR, Odds Ratio; PAI, plasminogen activator inhibitor- I; CI, Confidence Interval; LCL, Lower Confidence Level; UCL, Upper Confidence Level.

\section{Discussion}

Recent studies have suggested that bone loss in postmenopausal women is mediated, at least in part, by increased production of certain cytokines. ${ }^{2,3,28,29}$ We recently reported that mitogen-stimulated peripheral blood mononuclear cells
(PBMCs) from women with low BMD produced significantly higher levels of the pro-resorptive cytokines TNF- $\alpha$, IL-6, IL-12 and IL-17 and lower levels of the anti-resorptive cytokines IL-4, IL10, IL-13 and IL-23 as compared to women with normal BMD. Moreover, the pro-/anti- 
resorptive cytokine ratios further suggested a stronger systemic bias towards stimulators of resorptive osteoclastogenesis that is lowest in women with normal BMD, modest in women with osteopenia and highest in women with osteoporosis. ${ }^{28}$ We expected that this pattern will be reflected in the circulatory plasma levels of these patients. However, this was not the case as none of the circulatory levels of the cytokines tested showed significant difference between the groups. This is further in line with our earlier finding that cytokine production patterns of peripheral blood mononuclear cells are more discriminatory than plasma measurements of cytokine profiles. ${ }^{30}$

Cytokines have paracrine and autocrine effects, and are generally short-range factors that act locally. However, using their circulatory levels as a marker for diseases has been controversial. Taking rheumatoid arthritis (RA) as an example, studies have reported a range from no detectable levels of cytokines in sera or plasma, to no significant differences between the healthy and RA groups and up to a wide range of significant differences in a variable number of individual or combinations of cytokines (reviewed $\mathrm{in}^{31}$ ). Few studies have measured serum levels of cytokines in relation to osteoporosis. As compared to postmenopausal women with normal BMD, age-matched osteoporotic women were reported to have higher serum levels IL-1 and IL-8, ${ }^{32}$ higher IL-17, lower IL-4 and IFN- $\gamma$ levels but no significant differences in IL-6, TNF- $\alpha$, IL-2 and IL-10. ${ }^{33}$ One should bear in mind that cytokines work in complex hierarchical regulatory networks and most of them show pleiotropic action on multiple different targets, making the full understanding of the net effect or balance very challenging. It is worth mentioning that serum levels may vary due to several patient factors such as age, gender, circadian rhythm, food intake, exercise and stress. ${ }^{31}$ It has further been suggested that measuring cytokines in plasma is more accurate than that in serum as the clotting process itself may contribute to cytokine production. ${ }^{34-36}$

It is interesting that circulatory levels of all adipokines were measurable and most were significantly different between the groups studied. The mechanisms underlying the associations between adipokines and bone health are poorly understood, and reports are controversial. While some researchers have reported a lack of correlation between adipokiens and bone mineral density, ${ }^{8}$ others pointed out that some (such as adiponectin) may be considered as a potential biomarker of osteoporosis in postmenopausal women, ${ }^{37,38}$ or even an independent predictor of BMD. ${ }^{7,39}$ Among the possible mechanisms suggested was a link between sex hormones, adiponectin metabolism, production of pro-inflammatory factors and menopause transition. ${ }^{40-42}$ Of the five adipokines we tested, adiponectin levels show significant negative correlation with BMD of the hip and spine, which supports a previous metanalysis. ${ }^{9}$ Further studies are needed to investigate the possible correlation between levels of serum adipokines and BMD in relation to other demographic parameters.

Scientific research still supports the potential of BTMs to provide clinical useful information although many of the identified limitations remain. ${ }^{18}$ This is further supported by a recent meta-analysis that showed modest but significant association between levels of PINP and CTX and risk of future fractures. ${ }^{15}$ Our work contributes into providing data from Gulf region using the standardized analytical methods of the reference analytes as recommended by the IOF-IFCC Bone Marker Standards Working Group. ${ }^{17}$ In the population sample studied, although circulatory levels of CTX were comparable, postmenopausal women with normal BMD had significantly higher levels of circulatory PINP and PINP/CTX ratio as compared to women with low BMD (Figure 2). Circulatory levels of PINP and PINP/CTX correlated significantly with BMD of the hip and spine.

We found estradiol levels to significantly correlate with circulatory levels of PINP, PINP/CTX ratio and BMD of the hip and spine. Furthermore, estradiol levels were significantly higher in postmenopausal women with normal BMD group as compared to women with low BMD. The rapid fall in serum levels of estrogen complicated by other comorbidities such as the low systemic inflammatory status associated with the onset of menopause, a phenomenon referred to as "inflamm-aging", 43 all may contribute to osteoporosis during menopause.

We further observed that circulatory levels of bone markers (PINP, PINP/CTX ratio) as well as adipokines markers (adiponectin, adipsin, PA-1 and resistin) were not affected by age. However, all markers, except adiponectin, were affected by years since menopause indicating that it is a confounding factor (Table 3). While this supports the overwhelming association between levels of estrogen and bone loss, large epidemiological studies have now demonstrated quite clearly that bone loss starts as early as in the early thirties and long before any changes in levels of sex steroids. ${ }^{19,44}$ Several researchers suggest that estrogen-deficiency in the pathogenesis of osteoporosis is incomplete; they also suggest that bone loss is due to 
several age-related factors which, in addition to changes in the ovaries, adrenal gland and kidney, is also due to other factors such as age-related oxidative stress, genetic predisposing factors as well as immune and inflammatory mediators. ${ }^{19,21,45}$ Collectively, the above lines research suggest that it would be appropriate to move from an estrogen-centric basis to aging and a multi-factorial context of the pathogenesis of osteoporosis. ${ }^{19,21}$

We realize that this study has several limitations. From a physiological point of view, although estimating circulating levels of cytokines and adipokines is less invasive and more practical, these may not reflect actual levels in tissues. Further, given that our dataset is based on only 71 women, and that the data have several variables, the findings and associations should be taken as tentative results, subject to future corroboration. Also, the data presented here do not prove causation. Having said that, our analysis and findings do point to several interesting avenues to be explored further.

In conclusion, our observations provide insights into the possible role of cytokines, adipokines and bone turnover markers on the pathogenesis of postmenopausal osteoporosis. This data may support future strategies for the prevention and/or reversal of systemic bone loss associated with osteoporosis, where the control of such markers may have diagnostic, therapeutic or monitoring value.

\section{Acknowledgments}

This study is supported by Kuwait Foundation of Advancement of Science (KFAS) projects no. 20131302-02 and PR17-18SL-01.

\section{Disclosure}

The authors declare that they have no conflicts of interest in this work.

\section{References}

1. Melton LJ 3rd, Chrischilles EA, Cooper C, Lane AW, Riggs BL. Perspective. How many women have osteoporosis? J Bone Miner Res. 1992;7(9):1005-1010. doi:10.1002/jbmr.5650070902

2. Malutan AM, Dan M, Nicolae C, Carmen M. Proinflammatory and anti-inflammatory cytokine changes related to menopause. Prz Menopauzalny. 2014;13(3):162-168. doi:10.5114/pm.2014.43818

3. Faienza MF, Ventura A, Marzano F, Cavallo L. Postmenopausal osteoporosis: the role of immune system cells. Clin Dev Immunol. 2013;2013:575936. doi:10.1155/2013/657424

4. Takayanagi H. Osteoimmunology and the effects of the immune system on bone. Nat Rev Rheumatol. 2009;5(12):667-676. doi:10.1038/ nrrheum.2009.217
5. Kapoor M, Martel-Pelletier J, Lajeunesse D, Pelletier JP, Fahmi H. Role of proinflammatory cytokines in the pathophysiology of osteoarthritis. Nat Rev Rheumatol. 2011;7(1):33-42. doi:10.1038/ nrrheum.2011.2

6. Pietschmann P, Mechtcheriakova D, Meshcheryakova A, FögerSamwald U, Ellinger I. Immunology of osteoporosis: a mini-review. Gerontology. 2015;62(2):128-137. doi:10.1159/000431091

7. Lubkowska A, Dobek A, Mieszkowski J, Garczynski W, Chlubek D. Adiponectin as a biomarker of osteoporosis in postmenopausal women: controversies. Dis Markers. 2014;2014:1-14. doi: 10.1155/ 2014/975178.

8. Mohiti-Ardekani J, Soleymani-Salehabadi H, Owlia MB, Mohiti A. Relationships between serum adipocyte hormones (adiponectin, leptin, resistin), bone mineral density and bone metabolic markers in osteoporosis patients. J Bone Miner Metab. 2014;32(4):400-404. doi:10.1007/s00774-013-0511-4

9. Biver E, Salliot C, Combescure C, et al. Influence of adipokines and Ghrelin on bone mineral density and fracture risk: a systematic review and meta-analysis. Journal Clin Endocrinol Metab. 2011;96 (9):2703-2713. doi:10.1210/jc.2011-0047

10. Jürimäe J, Jürimäe T, Leppik A, Kums T. The influence of ghrelin, adiponectin, and leptin on bone mineral density in healthy postmenopausal women. J Bone Miner Metab. 2008;26(6):618-623. doi:10.1007/s00774-008-0861-5

11. Mpalaris V, Anagnostis P, Anastasilakis AD, Goulis DG, Doumas A, Iakovou I. Serum leptin, adiponectin and ghrelin concentrations in post-menopausal women: is there an association with bone mineral density? Maturitas. 2016;88:32-36.

12. Tanna N, Patel K, Moore AE, Dulnoan D, Edwards S, Hampson G. The relationship between circulating adiponectin, leptin and vaspin with bone mineral density (BMD), arterial calcification and stiffness: a cross-sectional study in post-menopausal women. $J$ Endocrinol Invest. 2017;40(12):1345-1353. doi:10.1007/s40618-017-0711-1

13. Vosoberg K, Tillmann V, Tamm AL, Jurimae T, Maasalu K, Jurimae J. Adipocytokine and ghrelin levels in relation to bone mineral density in prepubertal rhythmic gymnasts entering puberty: a 3-year follow-up study. Eur J Appl Physiol. 2016;116(4):831-839. doi:10.1007/s00421-016-3338-6

14. Hlaing TT, Compston JE. Biochemical markers of bone turnoveruses and limitations. Ann Clin Biochem. 2014;51(2):189-202.

15. Johansson H, Odén A, Kanis JA, et al. A meta-analysis of reference markers of bone turnover for prediction of fracture. Calcif Tissue Int. 2014;94(5):560-567. doi:10.1007/s00223-014-9842-y

16. Bergmann P, Body JJ, Boonen S, et al. Evidence-based guidelines for the use of biochemical markers of bone turnover in the selection and monitoring of bisphosphonate treatment in osteoporosis: a consensus document of the Belgian bone club. Int $J$ Clin Pract. 2009;63(1):19-26. doi:10.1111/j.17421241.2008.01911.x

17. Vasikaran $S$, Eastell R, Bruyère $O$, et al. Markers of bone turnover for the prediction of fracture risk and monitoring of osteoporosis treatment: a need for international reference standards. Osteoporos Int. 2011;22(2):391-420. doi:10.1007/s00198-010-1501-1

18. Morris H, Eastell R, Jorgesen N, et al. Clinical usefulness of bone turnover marker concentrations in osteoporosis. Clin Chimica Acta. 2017;467:34-41.

19. Manolagas SC. From estrogen-centric to aging and oxidative stress: a revised perspective of the pathogenesis of osteoporosis. Endocr Rev. 2010;31(3):266-300. doi:10.1210/er.2009-0024

20. Almeida M, Laurent MR, Dubois V, et al. Estrogens and androgens in skeletal physiology and pathophysiology. Physiol Rev. 2017;97 (1):135-187. doi:10.1152/physrev.00033.2015 
21. Pouresmaeili F, Kamalidehghan B, Kamarehei M, Goh YM. A comprehensive overview on osteoporosis and its risk factors. Ther Clin Risk Manag. 2018;14:2029-2049. doi:10.2147/TCRM.S138000

22. Khosla S, Melton LJ 3rd, Riggs BL. Clinical review 144: estrogen and the male skeleton. J Clin Endocrinol Metab. 2002;87 (4):1443-1450. doi:10.1210/jcem.87.4.8417

23. Carani C, Qin K, Simoni M, et al. Effect of testosterone and estradiol in a man with aromatase deficiency. $N$ Engl $J$ Med. 1997;337 (2):91-95. doi:10.1056/NEJM199707103370204

24. Leder BZ, LeBlanc KM, Schoenfeld DA, Eastell R, Finkelstein JS. Differential effects of androgens and estrogens on bone turnover in normal men. J Clin Endocrinol Metab. 2003;88(1):204-210. doi:10.1210/jc.2002-021036

25. Hofbauer LC, Hicok KC, Chen D, Khosla S. Regulation of osteoprotegerin production by androgens and anti-androgens in human osteoblastic lineage cells. Eur J Endocrinol. 2002;147(2):269-273. doi:10.1530/eje.0.1470269

26. Hofbauer LC, Khosla S, Dunstan CR, Lacey DL, Spelsberg TC, Riggs BL. Estrogen stimulates gene expression and protein production of osteoprotegerin in human osteoblastic cells. Endocrinology. 1999;140(9):4367-4370. doi:10.1210/endo.140.9.7131

27. Manolagas SC. Birth and death of bone cells: basic regulatory mechanisms and implications for the pathogenesis and treatment of osteoporosis. Endocr Rev. 2000;21(2):115-137.

28. Azizieh F, Raghupathy R, Shehab D, Al-Jarallah K, Gupta R. Cytokine profiles in osteoporosis suggest a proresorptive bias. Menopause. GME.0000000000000885

29. Rauner M, Sipos W, Pietschmann P. Osteoimmunology. Int Arch Allergy Immunol. 2007;143(1):31-48. doi:10.1159/000098223

30. Azizieh FY, Al Jarallah K, Shehab D, Gupta R, Dingle K, Raghupathy R. Patterns of circulatory and peripheral blood mononuclear cytokines in rheumatoid arthritis. Rheumatol Int. 2017;37 (10):1727-1734. doi:10.1007/s00296-017-3774-6

31. Burska A, Boissinot M, Ponchel F. Cytokines as biomarkers in rheumatoid arthritis. Mediators Inflamm. 2014;2014:545493. doi: $10.1155 / 2014 / 545493$

32. Sahin G, Ozturk C, Bagis S, Cimen OB, Erdogan C. Correlation of serum cytokine levels with axial bone mineral density. Singapore Med J. 2002;43(11):576-578.

33. Zhang J, Fu Q, Ren Z, et al. Changes of serum cytokines-related Th1/Th2/Th17 concentration in patients with postmenopausal osteoporosis. Gynecological Endocrinol. 2015;31(3):183-190. doi: $10.3109 / 09513590.2014 .975683$
34. Whiteside TL. Cytokines and cytokine measurements in a clinical laboratory. Clin Diagn Lab Immunol. 1994;1(3):257-260.

35. Tworoger SS, Hankinson SE. Collection, processing, and storage of biological samples in epidemiologic studies: sex hormones, carotenoids, inflammatory markers, and proteomics as examples. Cancer Epidemiol Biomarkers Prev. 2006;15(9):1578-1581. doi:10.1158/ 1055-9965.EPI-06-0629

36. Hosnijeh FS, Krop EJ, Portengen L, et al. Stability and reproducibility of simultaneously detected plasma and serum cytokine levels in asymptomatic subjects. Biomarkers. 2010;15(2):140-148. doi:10.3109/13547500903340570

37. Wang Q-P, Yang L, Li X-P, et al. Effects of 17 $\beta$-estradiol on adiponectin regulation of the expression of osteoprotegerin and receptor activator of nuclear factor- $\kappa \mathrm{B}$ ligand. Bone. 2012;51(3):515-523. doi:10.1016/j.bone.2012.05.011

38. Ozkurt B, Ozkurt ZN, Altay M, Aktekin CN, Caglayan O, Tabak Y. The relationship between serum adiponectin level and anthropometry, bone mass, osteoporotic fracture risk in postmenopausal women. Eklem Hastalik Cerrahisi. 2009;20(2):78-84.

39. Wu N, Wang Q-P, Li H, Wu X-P, Sun Z-Q, Luo X-H. Relationships between serum adiponectin, leptin concentrations and bone mineral density, and bone biochemical markers in Chinese women. Clin Chimica Acta. 2010;411(9):771-775. doi:10.1016/j.cca.2010.02.064

40. Miyatani Y, Yasui T, Uemura H, et al. Associations of circulating adiponectin with estradiol and monocyte chemotactic protein-1 in postmenopausal women. Menopause. 2008;15(3):536-541. doi:10.1097/gme.0b013e31815c85ed

41. Tworoger SS, Mantzoros C, Hankinson SE. Relationship of plasma adiponectin with sex hormone and insulin-like growth factor levels. Obesity. 2007;15(9):2217-2224. doi:10.1038/oby.2007.535

42. Siemińska L, Cichoń-Lenart A, Kajdaniuk D, et al. [Sex hormones and adipocytokines in postmenopausal women]. Polski merkuriusz lekarski. 2006;20(120):727-730.

43. Franceschi C, Campisi J. Chronic inflammation (inflammaging) and its potential contribution to age-associated diseases. J Gerontol Series A. 2014;69(Suppl 1):S4-S9. doi:10.1093/gerona/glu057

44. Riggs BL, Melton LJ, Robb RA, et al. A population-based assessment of rates of bone loss at multiple skeletal sites: evidence for substantial trabecular bone loss in young adult women and men. J Bone Mineral Res. 2008;23(2):205-214. doi:10.1359/jbmr.071020

45. Parfitt A. Osteoporosis: 50 years of change, mostly in the right direction. In: Osteoporosis and Bone Biology. Compston J, Ralston $\mathrm{S}$, editors. London: The State of the Art International Medical Press; 2000:1-13.
Journal of Inflammation Research

\section{Publish your work in this journal}

The Journal of Inflammation Research is an international, peerreviewed open-access journal that welcomes laboratory and clinical findings on the molecular basis, cell biology and pharmacology of inflammation including original research, reviews, symposium reports, hypothesis formation and commentaries on: acute/chronic inflammation; mediators of inflammation; cellular processes; molecular mechanisms; pharmacology and novel anti-inflammatory drugs; clinical conditions involving inflammation. The manuscript management system is completely online and includes a very quick and fair peerreview system. Visit http://www.dovepress.com/testimonials.php to read real quotes from published authors. 